\title{
Computation-Aided Classical-Quantum Multiple Access to Boost Network Communication Speeds
}

\author{
Masahito Hayashi® ${ }^{1,2,3, \dagger}$ and Ángeles Vázquez-Castro $\odot^{4, *}$ \\ ${ }^{1}$ Shenzhen Institute for Quantum Science and Engineering, Southern University of Science and Technology, \\ Shenzhen 518055, China \\ ${ }^{2}$ Guangdong Provincial Key Laboratory of Quantum Science and Engineering, Southern University of Science and \\ Technology, Shenzhen 518055, China \\ ${ }^{3}$ Graduate School of Mathematics, Nagoya University, Nagoya 464-8602, Japan \\ ${ }^{4}$ Department of Telecommunications and Systems Engineering, Centre for Space Research (CERES) of Institut \\ d'Estudis Espacials de Catalunya (IEEC-UAB), Universitat Autònoma de Barcelona, Bellaterra (Barcelona)
} 08193, Spain

(Received 3 July 2021; revised 13 July 2021; accepted 4 October 2021; published 10 November 2021)

\begin{abstract}
A multiple access channel (MAC) consists of multiple senders simultaneously transmitting their messages to a single receiver. For the classical-quantum case (CQ MAC), achievable rates are known assuming that all the messages are decoded, a common assumption in quantum network design. However, such a conventional design approach ignores the global network structure, i.e., the network topology. When a CQ MAC is given as a part of quantum network communication, this work shows that computation properties can be used to boost communication speeds with code design dependent on the network topology. We quantify achievable quantum communication rates of codes with the computation property for a two-sender CQ MAC. When the two-sender CQ MAC is a boson coherent channel with binary discrete modulation, we show that it achieves the maximum possible communication rate (the single-user capacity), which cannot be achieved with conventional design. Further, such a rate can be achieved by different detection methods: quantum (with and without quantum memory), on-off photon counting, and homodyne (each at different photon power). Finally, we describe two practical applications, one of which cryptographic.
\end{abstract}

DOI: 10.1103/PhysRevApplied.16.054021

\section{INTRODUCTION}

Recently, quantum communication has been actively studied [1-4]. For practical use of quantum communication among many users, we need to establish large quantum networks involving a considerable number of nodes [5-12]. However, many of their theoretical studies do not reflect the network topology. To see this, as a typical part of a network, we focus on a two-user multiple access channel (MAC). It consists of two spatially separated senders aiming to transmit simultaneously their information messages to a single receiver. It appears in the uplink from many terrestrial terminals to an aerial or satellite node. Hence, designing a code for MAC, i.e., a MAC code, is essential for building a network. Existing studies investigated MAC codes for classical-quantum MAC (CQ MAC) and clarified the capacity region [13]. That is, these studies maximized the transmission rates under the conventional assumption that all messages from

\footnotetext{
*angeles.vazquez@uab.es

†hayashi@sustech.edu.cn
}

multiple senders are decoded. We call codes under such conventional assumption simultaneously decodable (SD) codes.

In general, the network topology may include bottlenecks, in which case, the information flow requires to be network-coded to achieve the network capacity as shown by Ahlswede et al. [14]. Since a CQ MAC is composed of a quantum signal from two senders, which arrive to only one receiver, as shown later, it becomes a bottleneck of a quantum network dependently on its topology. In this case, to boost the communication rates over the network, we need to resolve the bottleneck, which require a network code that takes into account the network topology (which SD codes do not consider). However, while several studies consider quantum network coding for noiseless channels under several topological assumptions [15-25], no existing results apply the network coding concept to the CQ MAC composed of imperfect channels, including lossy channels dependent on the network topology.

Here, to see how conventional codes underperform (i.e., do not achieve capacity) due to the bottleneck of the CQ $\mathrm{MAC}$ on a concrete network, we focus on the quantum 
one-hop relay network model, which is a simple, yet relevant network topology, and is composed of a relay node (e.g., aerial vehicles or satellites) in addition to two senders $A$ and $B$ (see Fig. 1). The two senders $A$ and $B$ are located in distant places so that they cannot directly communicate to each other. They can access only to the relay node (illustrated as a satellite in Fig. 1). Thus, this network model has two types of channels. One is the uplink, a CQ MAC (the network bottleneck) that has two senders $A$ and $B$ and one receiver, the relay node. The other is the downlink that is composed of two point-to-point CQ channels from the relay node to each of $A$ and $B$. The aim of this network is that the two senders $A$ and $B$ exchange their messages $M_{A}$ and $M_{B}$ via the relay. Although the use of CQ MAC saves time if compared to nonsimultaneous transmission, the quantum signal transmitted from one sender behaves as a noise for the quantum signal from another sender, which is the weak point of CQ MAC. Since the point-to-point CQ channels in the downlink have no such weak point, only the uplink is the bottleneck of the quantum one-hop relay network model. Note that, physically, when we employ the bosonic quantized electromagnetic fields, the CQ MAC in the uplink is given as interference of electromagnetic fields.

In the conventional relying method, the receiver at the relay decodes both messages in the uplink, and sends $M_{A}$ to sender $B$ and message $M_{B}$ to sender $A$ in the downlink. This relying method employs a SD code in the uplink, and is called direct forward (DF) because the relay directly decodes and forwards the messages. However, the relay can operate in a different way. To show a different relaying method to resolve the topological bottleneck, we need to introduce COMP codes over the CQ MAC. The operation of COMP codes compared to SD codes in a CQ MAC is illustrated in Fig. 2.

On the left, the use of COMP codes is shown when two senders transmit their messages $M_{A}$ and $M_{B}$ over a CQ MAC but only the modulo sum $M_{A} \oplus M_{B}$ is required to be reliably decoded. For comparison, the conventional SD codes in Refs. [13,26,27] is shown on the right, where the two messages $M_{A}$ and $M_{B}$ would be decoded simultaneously.

We observe that in principle, either SD or COMP codes can be used in our quantum one-hop network model. In other words, the satellite in Fig. 1 can be designed to use either SD or COMP codes. However, since the aim of the network is the exchange of messages between nodes $A$ and $B$, it is sufficient that the receiver at the relay node decodes only the modulo sum $M_{A} \oplus M_{B}$ in the uplink, where both messages are regarded as vectors on a finite field $\mathbb{F}_{p}$. This is because both senders decode the other's message by using the modulo operation after the relay broadcasts the recovered modulo sum $M_{A} \oplus M_{B}$ to both senders in the downlink. For example, sender $A$ can decode $M_{B}$ by $\left(M_{A} \oplus M_{B}\right) \ominus M_{A}$. In this paper, we use $\oplus$ and $\ominus$ to express the modulo plus and minus in the finite field $\mathbb{F}_{p}$ to distinguish it from the plus and minus in $\mathbb{C}$, respectively. This method employs a COMP code in the uplink, and is called computation-and-forward (CAF) strategy because the relay forwards the result of the computation of the messages. In the satellite network model in Fig. 2, our CQ MAC is given over quantized electromagnetic fields, a typical example of a bosonic system, and this method boosts the capacity by harnessing photon energy from the natural computation properties existing in the coherent interference of quantized electromagnetic fields.
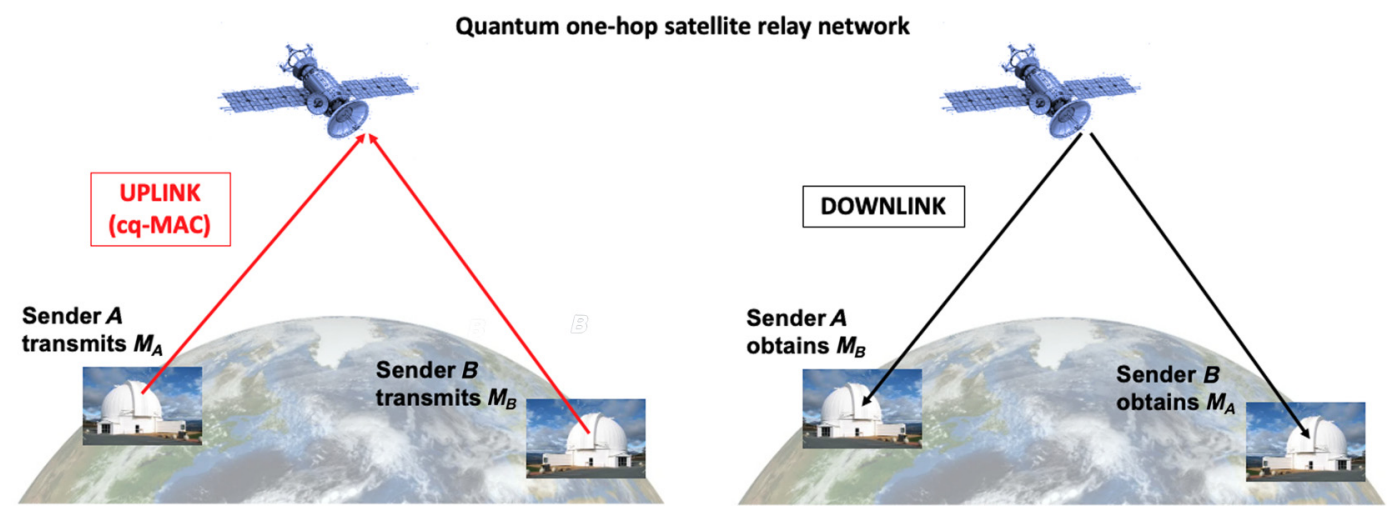

FIG. 1. Physical example of quantum one-hop relay network model. In this example, the relay is a satellite. The two senders $A$ and $B$ are located in distant places so that they cannot directly communicate to each other. In phase 1 , the two senders access simultaneously the satellite. Hence, the uplink is a CQ MAC (the network bottleneck) that has two senders $A$ and $B$ and a single decoding system on the satellite. In phase 2 , the relay communicates to each sender via two point-to-point CQ channels towards senders $A$ and $B$. In this work we show that conventional decoding is not optimal (i.e., cannot achieve the capacity of the network) and introduce computation (COMP) codes that allow the actual sum of (quantized) electromagnetic fields in the CQ MAC of phase 1 to be decoded, enabling network capacity to be achieved. 

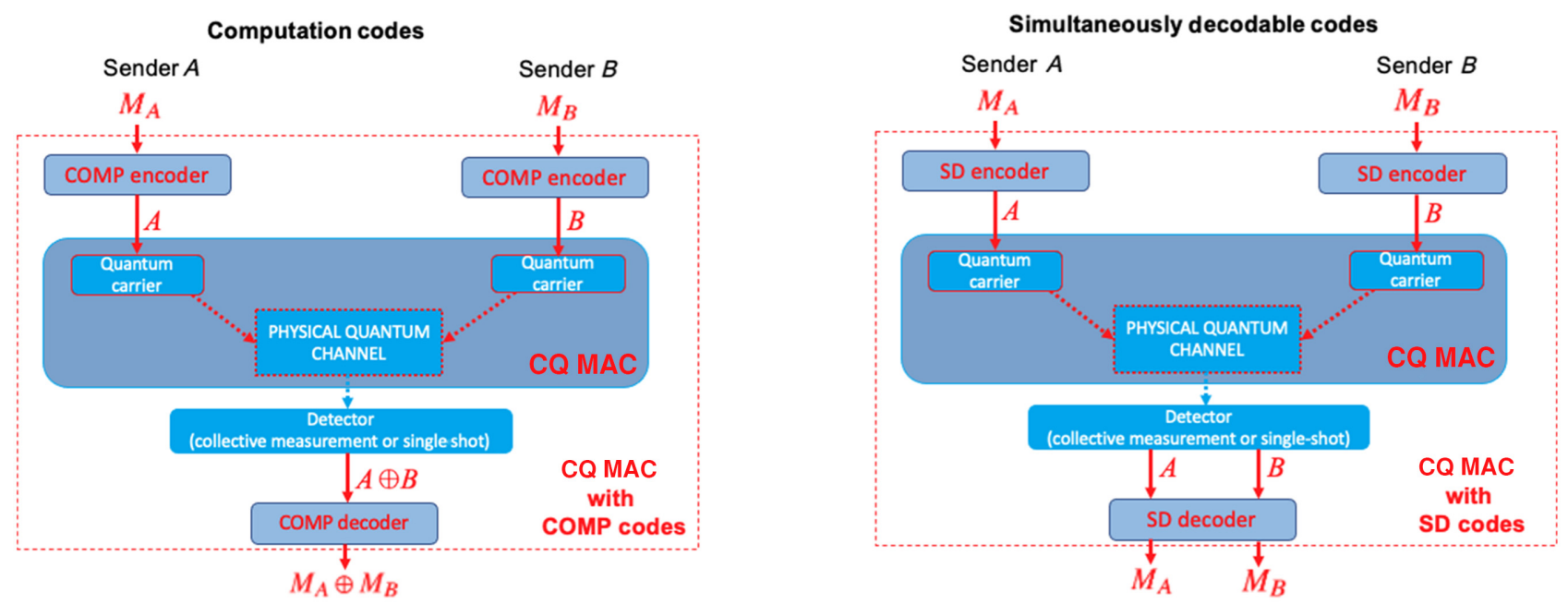

FIG. 2. Illustration of the operation of COMP codes proposed in this work, compared to conventional SD codes when two senders simultaneously transmitting their messages over a CQ MAC. The diagram on the left illustrates the concept of COMP codes. The main objective of our work is the design of the required COMP codes when only the modulo sum $M_{A} \oplus M_{B}$ needs to be reliably transmitted over the CQ MAC. For comparison, the conventional approach $[13,26]$ is shown on the right: the receiving node decodes both messages, $M_{A}$ and $M_{B}$ using conventional simultaneous-decodable codes, denoted here as SD codes.

In the classical case, our proposed type of codes are said to provide reliable physical-layer network coding because the code is directly applied to the physical signal naturally combined in the channel. However, while our proposed strategy seemingly corresponds to the quantum analog of physical-layer network coding (which has been extensively studied [28-39]), in fact it does not. The reason is that in traditional classical networks and the Internet, physical networks and their logical abstractions are purposely separately designed and managed (e.g., the former by engineers the latter by computer scientists). Fully quantum networks, however, no longer have such separation because its developing stage is still in the pioneer days before establishing the division between physical and logical parts. To extract the performance of the quantum network, we need to seamlessly design the communication method over the quantum network.

In summary, the main issue is a study on the codes for a CQ MAC that take into account the network topology, i.e., COMP codes for a CQ MAC. The remaining part of this paper is organized as follows. In Sec. II, we obtain a lower bound of the achievable rate of COMP codes over a general CQ MAC as Theorem 1. Theorem 1 is shown in Appendix B after a required preparation for analysis on affine codes in Appendix A. In Sec. III, we then apply them over CQ MAC with boson coherent states $[26,27]$ and quantify the gains in reliable rates with respect to conventional SD codes for several detection strategies. We show that COMP codes boost communication rates by taking advantage of the computation properties inherent in the coherent quantum interference (of quantized electromagnetic fields). When our method is applied to quantized electromagnetic fields, COMP codes are interpreted to gather photon energy from interference instead of treating it as noise (like conventional codes do). In terms of relying strategy for our network of reference (Fig. 1), our results show that the CAF relaying strategy is preferred over the conventional DF strategy.

In Sec. IV, to show the usefulness of the seamless design, in addition to the CAF strategy, we introduce another significant application, which shows the wide applicability of our proposed COMP codes. For this aim, we abstract away a quantum physical server as a logical server node. We then show that COMP codes can be applied to symmetric private information retrieval with such servers. Private information retrieval (PIR) with multiple servers is a method for a user to download a file from noncommunicating servers each of which contains the copy of a classical file set while the identity of the downloaded file is not leaked to each server. Symmetric PIR (SPIR) with multiple servers achieves the above task without leakage of other files to the user. Hence, this is another contribution of our work as a similar result is not yet existing in the literature, which has only treated noiseless channels $[40,41]$. In Sec. V, although the merit of COMP codes depends on the network topology, there are many other types of topologies whose bottlenecks can be resolved by our method, such as the well-known butterfly network [14-16,22].

\section{ACHIEVABLE RATES OF COMP CODES}

For simplicity, we assume the two senders of the CQ MAC, denoted as $\mathcal{W}$, having the same codebook with the alphabets $\mathcal{A}$ and $\mathcal{B}$ defined in $\mathbb{F}_{p}$. Each message $M_{k}^{n}, k=$ $A, B$ is assumed to be chosen independently and uniformly 
from $\mathcal{M}_{k}^{n}$. We define a $\left(2^{n R}, n\right)$ COMP code as consisting of two message sets that are equivalent to $\mathcal{M}^{n}=\mathbb{F}_{p}^{\ell}$ with $\left|\mathbb{F}_{p}^{\ell}\right| \cong\left[2^{n R}\right]$. Two encoders $\phi_{1}^{n}$ and $\phi_{2}^{n}$ map each message $M_{k}^{n} \in \mathcal{M}^{n}$ to a sequence $\phi_{k}^{n}\left(M_{k}^{n}\right) \in \mathbb{C}^{n}$ and $k=1,2$. Then, after measuring the received quantum system, the receiver estimates a linear combination of $M_{1}^{n} \oplus M_{2}^{n} \in \mathcal{M}^{n}$.

The rate $R$ is achievable if there exists a sequence of $\left(2^{n R}, n\right)$ computation codes such that $\lim _{n \rightarrow \infty} P_{\text {COMP }}^{n}=$ 0 , with $P_{\text {COMP }}^{n}$ the error probability. The supremum of achievable rates for computation is called the computation rate and is denoted by $C_{\text {COMP. }}$ To get its lower bound instead of $C_{\mathrm{COMP}}(\mathcal{W})$, we denote by $I(A \oplus B ; Y)_{P_{U} \times P_{U}}$ the mutual information when $A$ and $B$ are subject to the uniform distribution $P_{U}$ independently, i.e., $P_{A} \times P_{B}=P_{U} \times$ $P_{U}$. The capacity $C_{\mathrm{COMP}}(\mathcal{W})$ is evaluated in the following theorem.

Theorem 1. We have the following lower and upper bounds of $C_{\mathrm{COMP}}(\mathcal{W})$.

$$
\begin{gathered}
\underline{C}_{\mathrm{COMP}}(\mathcal{W}):=I(A \oplus B ; Y)_{P_{U} \times P_{U}} \leq C_{\mathrm{COMP}}(\mathcal{W}) \\
\bar{C}_{\mathrm{COMP}}(\mathcal{W}) \\
:=\min \left(\max _{P_{A}, b \in \mathbb{F}_{p}} I(A ; Y \mid B=b)_{P_{A}},\right. \\
\left.\max _{P_{B}, a \in \mathbb{F}_{p}} I(B ; Y \mid A=a)_{P_{B}}\right) \\
\geq C_{\mathrm{COMP}}(\mathcal{W}),
\end{gathered}
$$

where the distribution of $A$ and $B$ are limited to the uniform distribution in Eq. (1) and the maximum in Eq. (2) is taken among distributions $P_{A}$ or $P_{B}$ of $A$ or $B$.

Theorem 1 is proved in Appendix B by combining the method for degraded channel [36], the operator inequality developed in Ref. [42], the technique based on the Toeplitz matrix [43], and affine codes. Note that a conventional SD code is defined as a $\left(2^{n R_{A}}, 2^{n R_{B}}, n\right)$ with two message sets $\left[2^{n R_{k}}\right], k=A, B$ and two encoders $\phi_{1}^{n}$ and $\phi_{2}^{n}$, which use different codes to map each message $M_{k}^{n} \in$ $\left[2^{n R_{k}}\right]$ to a sequence $\phi_{k}^{n}\left(M_{k}^{n}\right) \in \mathbb{C}^{n}, k=A, B$. Each message $M_{k}^{n}, k=A, B$ is assumed to be chosen independently from $\left[2^{n R_{k}}\right]$. In this case, the receiver outputs two estimations, of $M_{A}^{n}$ and of $M_{B}^{n}$. The pair $\left(R_{A}, R_{B}\right)$ is achievable for simultaneous-decodable code if there exists a sequence of $\left(2^{n R_{A}}, 2^{n R_{B}}, n\right)$ simultaneous-decodable codes such that $\lim _{n \rightarrow \infty} P_{\mathrm{SD}}^{n}=0$, where $P_{\mathrm{SD}}^{n}$ is the error probability. In this case, the capacity region for $\mathrm{SD}$ codes, $\mathcal{C}(\mathcal{W})$, were obtained by Yen and Shapiro [26] and Winter [13] treating interference as noise. When a SD code is used, the rate is limited as

$$
\begin{aligned}
C_{\mathrm{SD}}(\mathcal{W}):= & \max _{\left(R_{A}, R_{B}\right) \in \mathcal{C}(\mathcal{W})} \min \left(R_{A}, R_{B}\right) \\
= & \max _{P_{A} \times P_{B}} \min \left(I(A ; Y \mid B)_{P_{A} \times P_{B}}, I(B ; Y \mid A)_{P_{A} \times P_{B}},\right. \\
& \left.\frac{1}{2} I(A B ; Y)_{P_{A} \times P_{B}}\right) .
\end{aligned}
$$

In fact, to achieve the rates $\underline{C}_{\mathrm{COMP}}(\mathcal{W})$ and $C_{\mathrm{SD}}(\mathcal{W})$, the decoder needs to use collective measurement across $n$ receiving signal states. This measurement requires the technologies to store many receiving quantum signal states and perform a quantum measurement across many quantum signal states. To avoid use of quantum memory, the receiver needs to measure the receiving states individually.

As a simple case, we consider the situation where the receiver applies the same measurement $\Pi$ to all receiving signals. We denote the measurement outcome by $Z$. Then, we can define the mutual information $I(A \oplus B ; Z)_{\Pi}$. It is natural that the receiver's decoding operation is limited to the classical data processing over the collection of the outcomes of the same measurement $\Pi$. This method does not require quantum memory. In this case, we have the following achievable rate by using the result of classical COMP codes:

$$
\underline{C}_{\mathrm{COMP}, \Pi}(\mathcal{W}):=I(A \oplus B ; Z)_{\Pi, P_{U} \times P_{U}} .
$$

Optimizing the choice of the measurement, we obtain the rate $\underline{C}_{\mathrm{COMP}}^{c}(\mathcal{W}):=\max _{\Pi} \underline{C}_{\mathrm{COMP}, \Pi}(\mathcal{W})$ achieved without quantum memory. Besides, the inequality $\underline{C}_{\mathrm{COMP}}^{c}(\mathcal{W}) \leq$ $\underline{C}_{\text {COMP }}(\mathcal{W})$ holds, the equality holds if and only if the density matrices $\left\{(1 / p) \sum_{a \in \mathbb{F}_{p}} W_{a, b \ominus a}\right\}_{b \in \mathbb{F} p}$ are commutative with each other. Hence, in this case the receiver does not need to use collective measurement across $n$ receiving signal states. When a SD code is used and the receiver applies the measurements $\Pi$ to all receiving signal states, the rate is limited as

$$
\begin{gathered}
C_{\mathrm{SD}, \Pi}(\mathcal{W}):=\max _{P_{A} \times P_{B}} \min \left(I(A ; Z \mid B)_{\Pi, P_{A} \times P_{B}},\right. \\
\left.I(B ; Z \mid A)_{\Pi, P_{A} \times P_{B}}, \frac{1}{2} I(A B ; Z)_{\Pi, P_{A} \times P_{B}}\right) .
\end{gathered}
$$

Optimizing the choice of the measurement, we obtain the rate $C_{\mathrm{SD}}^{c}(\mathcal{W}):=\max _{\Pi} C_{\mathrm{SD}, \Pi}(\mathcal{W})$.

\section{APPLICATION TO BPSK DISCRETE MODULATION OVER LOSSY BOSONIC CHANNEL}

\section{A. Formulation of BPSK discrete modulation over lossy bosonic channel}

As a practical application of our results, we now show the application of COMP codes over boson coherent channels of optical coherent light. It corresponds to a realistic 
scenario where multiple sending devices communicate with a single receiver (terrestrial, aerial, or space borne). In this case, physically, COMP codes match computation properties of (quantized) physical electromagnetic carriers. For a feasible implementation, we focus on the practical discrete modulation binary phase-shift keying (BPSK). BPSK is obtained by choosing the input complex amplitudes of senders $A$ and $B$ to be $(-1)^{a} \tau^{-1 / 2} \alpha$ and $(-1)^{b} \tau^{-1 / 2} \alpha$, respectively, for $a, b \in\{0,1\}$, where the photon-number constraint at each sender is given as $|\alpha|^{2}, \tau$ is the transmittance for modes $A$ and $B$ of the channel, which we assume equal in both channels. More precisely, denoting the signal modes as $\hat{a}_{A}, \hat{a}_{B}$ for senders $A$ and $B$, respectively and $\hat{a}_{E}$ the environment mode, the receiver's mode $\hat{a}_{R}$ is given as $[26,27,44]$

$$
\hat{a}_{R}=\sqrt{\tau} \hat{a}_{A}+\sqrt{\tau} \hat{a}_{B}+\sqrt{1-2 \tau} \hat{a}_{E} .
$$

Then, the receiver obtains the coherent state

$$
W_{a, b}:=\left|\left[(-1)^{a}+(-1)^{b}\right] \alpha\right\rangle\left\langle\left[(-1)^{a}+(-1)^{b}\right] \alpha\right| .
$$

We denote this CQ MAC as $\mathcal{W}_{\alpha}$. This channel can be implemented by controlling the photonic power of the input signals in both sender sides. Here, we explain only bounds with simple expressions. By using the constants $\tilde{c}_{0}:=\left(1+e^{-4|\alpha|^{2}}\right) / 2, a_{o \mid \alpha}:=e^{-|\alpha|^{2}} \sinh |\alpha|^{2}$, and $a_{\bar{e} \mid \alpha}:=e^{-|\alpha|^{2}}\left(\cosh |\alpha|^{2}-1\right)$, our upper and lower bounds $\bar{C}_{\mathrm{COMP}}\left(\mathcal{W}_{\alpha}\right)$ and $\underline{C}_{\mathrm{COMP}}\left(\mathcal{W}_{\alpha}\right)$ have the following simple analytical expressions:

$$
\begin{aligned}
& \bar{C}_{\mathrm{COMP}}\left(\mathcal{W}_{\alpha}\right)=h\left(a_{o \mid \alpha}\right) \\
& \underline{C}_{\mathrm{COMP}}\left(\mathcal{W}_{\alpha}\right) \\
& =H\left[\left(\begin{array}{ccc}
\tilde{c}_{0} & \sqrt{a_{\bar{e} \mid 2 \alpha}} e^{-2|\alpha|^{2}} / 2 & 0 \\
\sqrt{a_{\bar{e} \mid 2 \alpha}} e^{-2|\alpha|^{2}} / 2 & a_{\bar{e} \mid 2 \alpha} / 2 & 0 \\
0 & 0 & a_{o \mid 2 \alpha} / 2
\end{array}\right)\right] \\
& \quad-\frac{1}{2} h\left(a_{o \mid 2 \alpha}\right) .
\end{aligned}
$$

Also, the achievable rate by the on-off measurement is calculated as

$$
\underline{C}_{\mathrm{COMP}, \text { on-off }}\left(\mathcal{W}_{\alpha}\right)=h\left(\tilde{c}_{0}\right)-\frac{1}{2} h\left(e^{-4|\alpha|^{2}}\right),
$$

where $h$ is the binary entropy function and $H$ is the von Neumann entropy.

\section{B. Rate $C_{\mathrm{SD}}\left(\mathcal{W}_{\alpha}\right)$ for SD codes}

When we consider the conditional mutual information $I(A ; Y \mid B)$, we calculate the mutual information between the input $A$ and the output quantum system $Y$ under the condition that the variable $B$ is fixed to a certain value $b$. Hence, it is sufficient to calculate the mutual information for the channel $a \mapsto\left|\left[(-1)^{a}+(-1)^{b}\right] \alpha\right\rangle\left\langle\left[(-1)^{a}+(-1)^{b}\right] \alpha\right|$. Since $b$ is a fixed value, applying the displacement unitary $D\left[-(-1)^{b} \alpha\right]$, we have the state $\left|(-1)^{a} \alpha\right\rangle\left\langle(-1)^{a} \alpha\right|$. That is, it is sufficient to calculate the mutual information for the channel $a \mapsto\left|(-1)^{a} \alpha\right\rangle\left\langle(-1)^{a} \alpha\right|$. By using two orthogonal states

$$
\begin{aligned}
& \left|\operatorname{odd}_{\alpha}\right\rangle:=\left(\sinh |\alpha|^{2}\right)^{-1 / 2} \sum_{m=0}^{\infty} \frac{\alpha^{2 m+1}}{\sqrt{(2 m+1) !}}|2 m+1\rangle, \\
& \left|\operatorname{even}_{\alpha}\right\rangle:=\left(\cosh |\alpha|^{2}\right)^{-1 / 2} \sum_{m=0}^{\infty} \frac{\alpha^{2 m}}{\sqrt{(2 m) !}}|2 m\rangle,
\end{aligned}
$$

and $a_{o \mid \alpha}:=e^{-|\alpha|^{2}} \sinh |\alpha|^{2}$ and $a_{e \mid \alpha}:=e^{-|\alpha|^{2}} \cosh |\alpha|^{2}$, the coherent state is decomposed as

$$
| \pm \alpha\rangle=\sqrt{a_{e \mid \alpha}}\left|\operatorname{even}_{\alpha}\right\rangle \pm \sqrt{a_{o \mid \alpha}}\left|\operatorname{odd}_{\alpha}\right\rangle .
$$

Dependently of $P_{A}$ and $P_{B}$, we have

$$
\begin{aligned}
& I(A ; Y \mid B)=H\left[P_{A}(0)|\alpha\rangle\left\langle\alpha\left|+P_{A}(1)\right|-\alpha\right\rangle\langle-\alpha|\right] \\
& \quad=H\left[\left(\begin{array}{cc}
a_{o \mid \alpha} & c\left[\alpha, P_{A}(0)\right] \\
c\left[\alpha, P_{A}(0)\right] & a_{e \mid \alpha}
\end{array}\right)\right]
\end{aligned}
$$

where $c\left[\alpha, P_{A}(0)\right]:=\left[1-2 P_{A}(0)\right] \sqrt{a_{o \mid \alpha} a_{e \mid \alpha}}$. In particular, when $P_{A}(0)=P_{A}(1)=1 / 2$, the off-diagonal part is zero. Hence, we have

$$
H\left[\frac{1}{2}|\alpha\rangle\left\langle\alpha\left|+\frac{1}{2}\right|-\alpha\right\rangle\langle-\alpha|\right]=h\left(a_{o \mid \alpha}\right) .
$$

For the calculation of $I(A B ; Y)$, we need to handle three states $|0\rangle,|2 \alpha\rangle,|-2 \alpha\rangle$. For this aim, instead of $\left|\operatorname{even}_{\alpha}\right\rangle$, we introduce another state:

$$
\left|\overline{\operatorname{even}}_{\alpha}\right\rangle:=a_{\bar{e} \mid \alpha}^{-1 / 2} e^{-|\alpha|^{2} / 2} \sum_{m=1}^{\infty} \frac{\alpha^{2 m}}{\sqrt{(2 m) !}}|2 m\rangle,
$$

where $\quad a_{\bar{e} \mid \alpha}:=e^{-|\alpha|^{2}}\left(\cosh |\alpha|^{2}-1\right)=e^{-|\alpha|^{2}} \sum_{m=1}^{\infty} \frac{|\alpha|^{4 m}}{(2 m) !}$. The state $\left|\overline{\operatorname{even}}_{\alpha}\right\rangle$ is orthogonal to $|0\rangle$ and $\left|\operatorname{odd}_{\alpha}\right\rangle$. The coherent state $|2 \alpha\rangle$ is decomposed in another way as

$$
| \pm 2 \alpha\rangle=e^{-2|\alpha|^{2}}|0\rangle+\sqrt{a_{\bar{e} \mid 2 \alpha}}\left|\overline{\operatorname{even}}_{2 \alpha}\right\rangle \pm \sqrt{a_{o \mid 2 \alpha}}\left|\operatorname{odd}_{2 \alpha}\right\rangle,
$$

Based on the basis $\left\{|0\rangle,\left|\operatorname{odd}_{2 \alpha}\right\rangle,\left|\overline{\operatorname{even}}_{2 \alpha}\right\rangle\right\}$, we find that

$$
\begin{aligned}
& I(A B ; Y) \\
&= H\left[P_{A}(0) P_{B}(0)|2 \alpha\rangle\left\langle 2 \alpha\left|+P_{A}(1) P_{B}(1)\right|-2 \alpha\right\rangle\langle-2 \alpha|\right. \\
&\left.+\left(P_{A}(0) P_{B}(1)+P_{A}(1) P_{B}(0)\right)|0\rangle\langle 0|\right] \\
&= H\left[\left(\begin{array}{ccc}
c_{0} & c_{2} & c_{3} \\
c_{2} & p_{A B} a_{o \mid 2 \alpha} & c_{1} \\
c_{3} & c_{1} & p_{A B} a_{\bar{e} \mid 2 \alpha}
\end{array}\right)\right]
\end{aligned}
$$


where

$$
\begin{aligned}
p_{A B} & :=P_{A}(0) P_{B}(0)+P_{A}(1) P_{B}(1), \\
c_{0} & :=\left[P_{A}(0) P_{B}(1)+P_{A}(1) P_{B}(0)\right]+p_{A B} e^{-4|\alpha|^{2}}, \\
c_{1} & :=\left[P_{A}(0) P_{B}(0)-P_{A}(1) P_{B}(1)\right] \sqrt{a_{\bar{e} \mid 2 \alpha} a_{o \mid 2 \alpha},} \\
c_{2} & :=\left[P_{A}(0) P_{B}(0)-P_{A}(1) P_{B}(1)\right] \sqrt{a_{o \mid 2 \alpha}} e^{-2|\alpha|^{2}}, \\
c_{3} & :=\left[P_{A}(0) P_{B}(0)+P_{A}(1) P_{B}(1)\right] \sqrt{a_{\bar{e} \mid 2 \alpha}} e^{-2|\alpha|^{2}} .
\end{aligned}
$$

Therefore, $I\left(\mathcal{W}_{\alpha}\right)$ is given as the following maximum:

$$
I\left(\mathcal{W}_{\alpha}\right)=\max _{P_{A}, P_{B}} H\left[\left(\begin{array}{ccc}
c_{0} & c_{2} & c_{3} \\
c_{2} & p_{A B} a_{o \mid 2 \alpha} & c_{1} \\
c_{3} & c_{1} & p_{A B} a_{\bar{e} \mid 2 \alpha}
\end{array}\right)\right] .
$$

Since $W_{0,1}$ and $W_{1,0}$ are the same state, the mutual information $I(A B ; Y)$ is upper bounded by $\log 3$. The three states $W_{0,1}=W_{1,0}, \quad W_{0,0}, \quad W_{1,1}$ can be distinguished when $\alpha$ is sufficiently large. Under this limit, the maximum of the mutual information $I(A B ; Y)$ converges to

$$
\begin{aligned}
I_{\max , \infty}:= & \max _{P_{A}, P_{B}} \eta\left(P_{A}(0) P_{B}(1)+P_{A}(1) P_{B}(0)\right) \\
& +\eta\left(P_{A}(0) P_{B}(0)\right)+\eta\left(P_{A}(1) P_{B}(1)\right),
\end{aligned}
$$

where $\eta(t):=-t \log t$. That is, under this limit, the transmission rate of SD codes converges to $I_{\max , \infty} / 2$.

\section{Rates for COMP codes}

In the setting with COMP codes, the lower bound of $C\left(\mathcal{W}_{\alpha}\right)$ given in Theorem 1 is calculated as

$$
\begin{aligned}
C\left(\mathcal{W}_{\alpha}\right) \geq & \underline{C}\left(\mathcal{W}_{\alpha}\right)=I(A \oplus B ; Y) \\
= & {\left[\left(\begin{array}{ccc}
\tilde{c}_{0} & 0 & \sqrt{a_{\bar{e} \mid 2 \alpha}} e^{-2|\alpha|^{2}} / 2 \\
0 & a_{o \mid 2 \alpha} / 2 & 0 \\
\sqrt{a_{\bar{e} \mid 2 \alpha}} e^{-2|\alpha|^{2}} / 2 & 0 & a_{\bar{e} \mid 2 \alpha} / 2
\end{array}\right)\right]-\frac{1}{2} H\left[\left(\begin{array}{ccc}
e^{-4|\alpha|^{2}} & 0 & \sqrt{a_{\bar{e} \mid 2 \alpha}} e^{-2|\alpha|^{2}} \\
0 & a_{o \mid 2 \alpha} & 0 \\
\sqrt{a_{\bar{e} \mid 2 \alpha}} e^{-2|\alpha|^{2}} & 0 & a_{\bar{e} \mid 2 \alpha}
\end{array}\right)\right] } \\
=H & {\left[\left(\begin{array}{ccc}
\tilde{c}_{0} & \sqrt{a_{\bar{e} \mid 2 \alpha}} e^{-2|\alpha|^{2}} / 2 & 0 \\
\sqrt{a_{\bar{e} \mid 2 \alpha}} e^{-2|\alpha|^{2}} / 2 & a_{\bar{e} \mid 2 \alpha} / 2 & 0 \\
0 & 0 & a_{o \mid 2 \alpha} / 2
\end{array}\right)\right]-\frac{1}{2} h\left(a_{o \mid 2 \alpha}\right) } \\
\geq H & {\left[\left(\begin{array}{ccc}
\tilde{c}_{0} & 0 & 0 \\
0 & a_{o \mid 2 \alpha} / 2 & 0 \\
0 & 0 & a_{\bar{e} \mid 2 \alpha} / 2
\end{array}\right)\right]-\frac{1}{2} H\left[\left(\begin{array}{ccc}
e^{-4|\alpha|^{2}} & 0 & 0 \\
0 & a_{o \mid 2 \alpha} & 0 \\
0 & 0 & a_{\bar{e} \mid 2 \alpha}
\end{array}\right)\right] } \\
= & h\left(\tilde{c}_{0}\right)-\frac{1}{2} h\left(e^{-4|\alpha|^{2}}\right) .
\end{aligned}
$$

Under the above relation, we consider the uniform distributions for $A$ and $B$. Hence, Eq. (8) follows from Eq. (19) while the exact value of the optimal transmission rate $C(\mathcal{W})$ is not known.

Since the three states $W_{0,1}=W_{1,0}, W_{0,0}, W_{1,1}$ can be distinguished when $\alpha$ is sufficiently large. Under this limit, the maximum of the mutual information $I(A \oplus B ; Y)$ converges to $\log 2$. Since $\log 2$ is the upper bound of $C(\mathcal{W})$, we can conclude that $C(\mathcal{W})$ converges to $\log 2$ under this limit.

Further, the rate $h\left(\tilde{c}_{0}\right)-\frac{1}{2} h\left(e^{-4|\alpha|^{2}}\right)$ can be achieved without use of collective measurement as follows. That is, it can be attained even with the following method. The receiver applies the on-off measurement to each signal state, and obtains the outcome $X$, where the on corresponds to 1 and the off does to 0 . The obtained channel is given as

$$
P_{X \mid A \oplus B}(0 \mid 0)=1, \quad P_{X \mid A \oplus B}(1 \mid 0)=0,
$$

$$
P_{X \mid A \oplus B}(0 \mid 1)=e^{-4|\alpha|^{2}}, \quad P_{X \mid A \oplus B}(1 \mid 1)=1-e^{-4|\alpha|^{2}}
$$

when $P_{A}$ and $P_{B}$ are the uniform distribution. Since the mutual information $I(A \oplus B ; X)$ equals $h\left(\tilde{c}_{0}\right)-$ $\frac{1}{2} h\left(e^{-4|\alpha|^{2}}\right)$, the rate $h\left(\tilde{c}_{0}\right)-\frac{1}{2} h\left(e^{-4|\alpha|^{2}}\right)$ can be attained by the combination of a classical encoder, a classical decoder, and the above on-off measurement. That is, this rate can be achieved with a feasible measurement without quantum memory, and hence, we obtain Eq. (9). 
Next, we discuss the upper bound $\bar{C}\left(\mathcal{W}_{\alpha}\right)$. We have

$$
\begin{aligned}
\max _{P_{B}, a \in \mathbb{F}_{2}} I(B ; Y \mid A=a)_{P_{B}} \\
=\max _{P_{B}, a \in \mathbb{F}_{2}} H\left[\sum_{b \in \in \mathbb{F}_{2}} P_{B}(b) \mid\left[(-1)^{a}\right.\right. \\
\left.\left.\left.\quad+(-1)^{b}\right] \alpha\right\rangle\left\langle\left[(-1)^{a}+(-1)^{b}\right] \alpha\right|\right] \\
=\max _{P_{B}} H\left[\sum_{b \in \in \mathbb{F}_{2}} P_{B}(b)\left|(-1)^{b} \alpha\right\rangle\left\langle(-1)^{b} \alpha\right|\right] \\
\stackrel{(a)}{=} \max _{P_{B}} H\left[\left(\begin{array}{cc}
a_{o \mid \alpha} & c\left[\alpha, P_{B}(0)\right] \\
c\left[\alpha, P_{B}(0)\right] & a_{e \mid \alpha}
\end{array}\right)\right] \\
=H\left[\left(\begin{array}{cc}
a_{o \mid \alpha} & 0 \\
0 & a_{e \mid \alpha}
\end{array}\right)\right]=h\left(a_{o \mid \alpha}\right),
\end{aligned}
$$

where (a) follows from Eq. (12). Notice that the quantity in the second line equals the single-sender Holevo quantity. In the same way, we have

$$
\max _{P_{A}, b \in \mathbb{F}_{2}} I(A ; Y \mid B=b)_{P_{A}}=H\left[\left(\begin{array}{cc}
a_{o \mid \alpha} & 0 \\
0 & a_{e \mid \alpha}
\end{array}\right)\right] .
$$

Thus, Eq. (7) holds.

\section{Case of conventional homodyne detection}

As a more feasible detection, we focus on conventional homodyne detection with BPSK that has an outcome in the continuous set $\mathbb{R}$. It corresponds to measuring one quadrature of the annihilation field operator in Eq. (5) with the outcome being the classical random variable defined as

$$
Y=\left[(-1)^{A}+(-1)^{B}\right]|\alpha|+Z,
$$

where $A$ and $B$ are input variables of senders $A$ and $B$, and $Z$ is the real-valued Gaussian variable with mean zero and variance $1 / 4$. In this case, the achievable rate of the COMP code is [36-38], [39, (17)]

$$
\begin{aligned}
I(A \oplus B ; Y) & =\tilde{H}(Y)-\tilde{H}(Y \mid A \oplus B) \\
& =h_{1}-\frac{1}{2} h_{2}-\frac{1}{4},
\end{aligned}
$$

where $\tilde{H}$ is the differential entropy, and $h_{1}$ and $h_{2}$ are defined by using $\eta(x)=-x \log x$ as

$$
\begin{aligned}
h_{1}:= & \int_{-\infty}^{\infty} \frac{\sqrt{2}}{\sqrt{\pi}} \eta\left(\frac{1}{4} e^{-2(y-2|\alpha|)^{2}}\right. \\
& \left.+\frac{1}{4} e^{-2(y+2|\alpha|)^{2}}+\frac{1}{2} e^{-2 y^{2}}\right) d y \\
h_{2}:= & \int_{-\infty}^{\infty} \frac{\sqrt{2}}{\sqrt{\pi}} \eta\left(\frac{1}{2} e^{-2(y-2|\alpha|)^{2}}+\frac{1}{2} e^{-2(y+2|\alpha|)^{2}}\right) d y .
\end{aligned}
$$

\section{E. Numerical analysis}

We numerically compare various analytically obtained results, which contains the rate with collective quantum detection and also the rates with photon counting (on-off) and homodyne detections. Since the collective quantum detection needs to be applied across multiple receiving quantum signal systems, it requires quantum memory. On the other hand, photon counting (on-off) and homodyne detections can be applied to a single receiving quantum signal system so that they do not need quantum memory.

Achievable rates in Fig. 3 (left) demonstrate the superiority of COMP codes for the bosonic CQ MAC network, as they achieve the upper bound of the capacity (1 bit per channel use for BPSK), while SD codes do not. Specifically, the rate of conventional codes with collective measurement, $C_{\mathrm{SD}}\left(W_{\alpha}\right)$, (which is maximum for uniform priors) achieves $I_{\max , \infty} / 2=[\eta(0.5)+\eta(0.25)+$ $\eta(0.25)] / 2=0.75$ bits per channel use at $|\alpha|^{2} \approx 1$, where $\eta(x)=-x \log _{2} x$. However, COMP codes with collective measurement achieve the transmission rate $\log _{2}(2)=1$ bits per channel use at $|\alpha|^{2} \approx 1$. Hence, COMP codes outperform conventional SD codes with gains up to $33.33 \%$ as shown in Fig. 3 (right), where we define gain between two rates, $R_{x}$ and $R_{y}$ as $100\left[\left(R_{x}-R_{y}\right) / R_{y}\right]$. In addition, the on-off measurement also achieves the transmission rate 1 with COMP codes but at a slower speed than with collective measurement, and it happens also at $|\alpha|^{2} \approx 1$.5. Even the conventional homodyne detection achieves the transmission rate 1 with COMP codes at $|\alpha|^{2} \approx 2$. The gains of our method for COMP codes over SD codes with collective measurement are positive only after a threshold photon power for any type of measurement. The thresholds of the collective measurement, the on-off measurement and the homodyne detection are $|\alpha|^{2}=0.18,0.45$, and 0.62 , respectively. The intuition of this result is that the quantum nature of the received signal offers an advantage for the decoding of the sum of the physical signal over SD codes above such threshold photon power while it does not below the threshold.

Notice that the collective quantum detection requires quantum memory because it needs to be applied across multiple receiving quantum signal systems. On the other hand, photon counting (on-off) and homodyne detections can be applied to a single receiving quantum signal system so that they do not need quantum memory. Therefore, COMP codes outperform SD codes even when COMP codes do not use quantum memory and SD codes use it.

As a useful reference, the single-sender Holevo quantity [i.e., $h\left(e^{-|\alpha|^{2}} \cosh |\alpha|^{2}\right)$ ] gives the upper bound, Eq. (2), of the capacity of the COMP code, as shown in Fig. 3 (left, green line). We observe that COMP codes with collective measurement is very close to the upper bound from a realistic photon average photon number of 1 . 

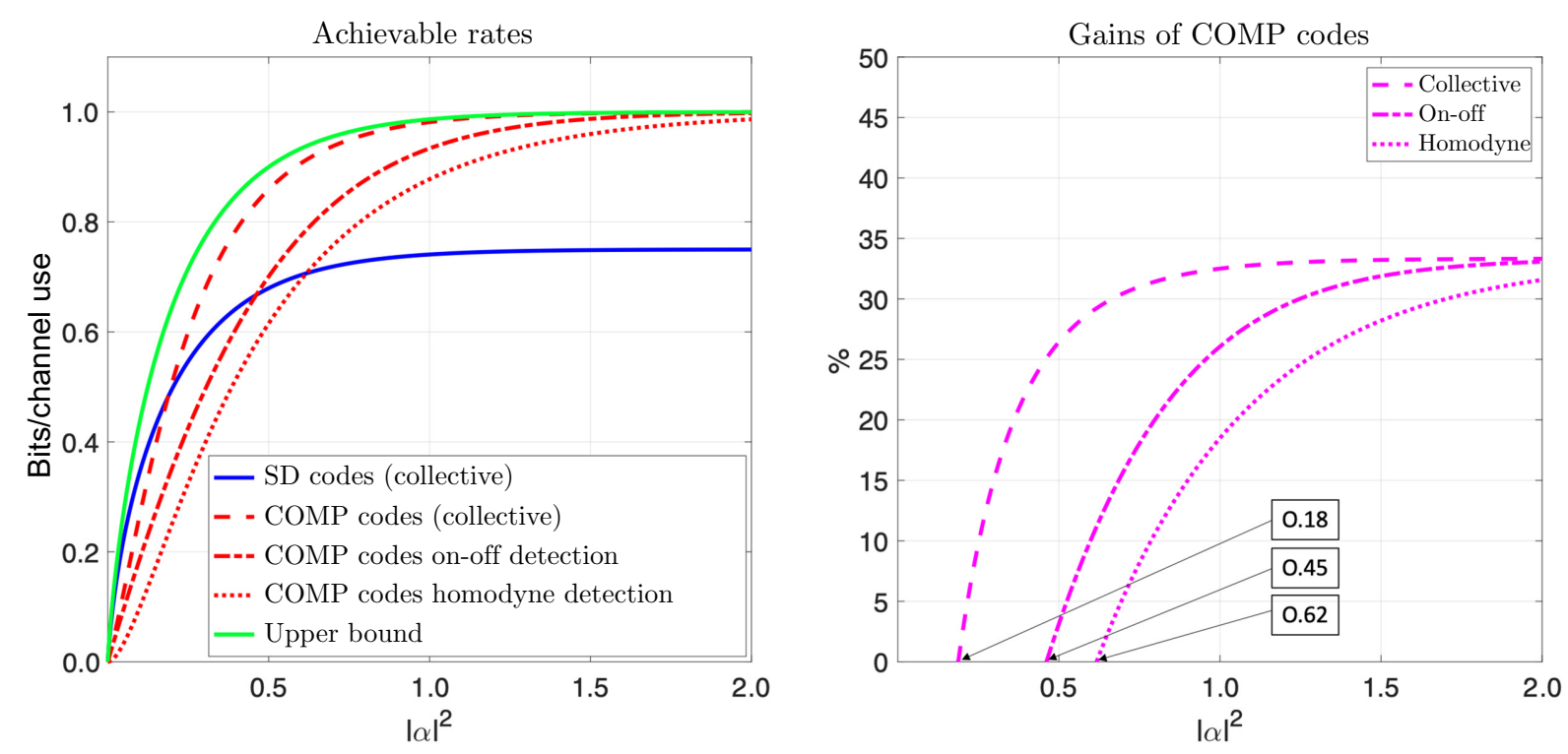

FIG. 3. Left: achievable communication rates with collective measurement, with SD codes, Eq. (17) (in blue) and with COMP codes for different measurements: collective as Eq. (19) (in red), on-off as Eq. (20) (in dashed red) and homodyne as Eq. (26) (in dashed point red). Right: the corresponding gains of COMP codes with different measurements over SD codes (up to 33.33\%). As a useful reference, the single-sender Holevo quantity [i.e. $\left.h\left(e^{-|\alpha|^{2}} \cosh |\alpha|^{2}\right)\right]$ gives the upper bound.

\section{APPLICATIONS TO QUANTUM NETWORKING TASKS}

Finally we show the practical relevance of our results describing the realistic application of COMP codes for two concrete quantum networking tasks: information exchange over the quantum one-hop relay network model and private information retrieval (SPIR protocol) between one user and two servers. While the former is the quantum analog of a classically known strategy as computation and forward, the latter is an application that has not been stated even in the classical setting, and therefore we provide a more detailed description.

\section{A. Information exchange between Alice and Bob}

Here, to clarify the difference from the application to SPIR, we summarize the CAF strategy. As explained in the Introduction, the CAF strategy assumes the quantum onehop relay network model, composed of a relay node (e.g., aerial vehicles or satellites) in addition to two senders, Bob and Alice, communicating over a wireless channel. The aim of this network is that Bob and Alice exchange their respective messages via the relay. Hence, it is sufficient that the receiver at the relay node decodes only the modulo sum $M_{A} \oplus M_{B}$ in the uplink, where both messages are regarded as vectors on a finite field $\mathbb{F}_{p}$. This is because both senders decode the other's message by using the modulo operation after the relay broadcasts the recovered modulo sum $M_{A} \oplus M_{B}$ to both senders by using two point-to-point channels. For example, sender $A$ can decode $M_{B}$ by $\left(M_{A} \oplus M_{B}\right) \ominus M_{A}$. This application is schematically illustrated in Fig. 4 (left). We observe that in-network computation (instead of simultaneous decoding) with COMP codes is enough to accomplish the networking task of information exchange between Bob and Alice.

\section{B. Private information retrieval}

Private information retrieval with multiple servers is a method for a user to download a file from noncommunicating servers each of which contains the copy of a classical file set while the identity of the downloaded file is not leaked to each server. This problem is trivially solved by requesting all files to one of the servers, but this method is inefficient. Also, this method allows the user to get information for other files that are not intended. Symmetric PIR with multiple servers is a protocol to achieve the above task without leakage of other files to the user. If the number of servers is one, this task is the same as the oblivious transfer, which is known as a difficult task. In a conventional setting, we assume that the number of servers and the number of files are fixed and all files have the same size and their sizes are asymptotically large. That is, the rate of the file size are increased linearly for the number $n$ of channel uses. In this case, the cost of upload from the user to the servers are negligible, i.e., the required information rate is zero. Only the size of download information is increased. Therefore, we optimize the maximum rate of the file size with respect to the number $n$ of channel uses.

When a classical noiseless channel from each server to the user is given and shared common random numbers 
Application for the quantum two-way relay network

Computation and forward

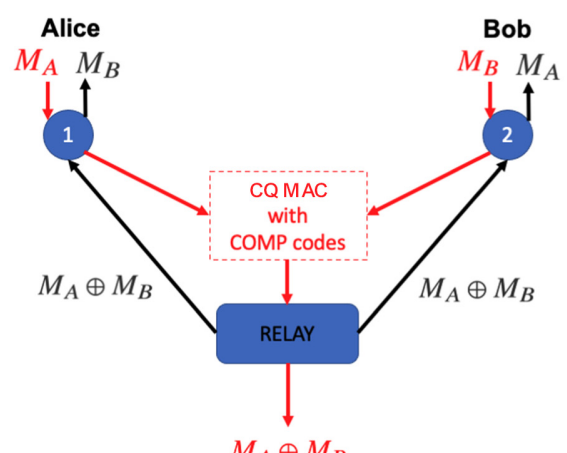

$M_{A} \oplus M_{B}$

Phase 1: uplink from Bob and Alice to the relay (CQ MAC) Phase 2: downlink towards Bob and Alice from the relay
Application for the quantum Internet network

Symmetric private information retrieval

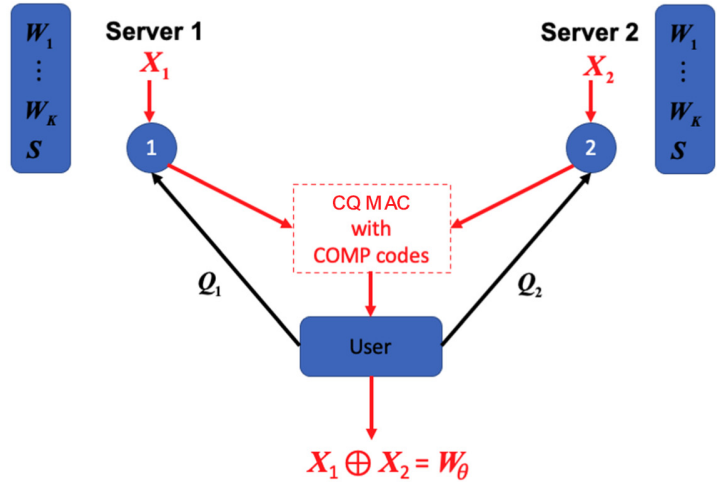

Phase 1: file querying from user to servers

Phase 2: (private) file retrieving from servers to user (CQ MAC)

FIG. 4. Left: schematic illustration of the quantum compute-and-forward relaying strategy, whereby Bob and Alice exchange their messages over a CQ MAC channel with COMP codes. Right: schematic illustration of our method for symmetric private information retrieval (SPIR) with two servers based on CQ MAC with COMP codes. Observe the very different role of the CQ MAC in the two applications while they employ the same network model, the quantum one-hop relay network model.

between servers are allowed, Ref. [40] derived the optimal protocol and its rate. Also, when a quantum noiseless channel from each server to the user is given and shared entangled states between servers are allowed, Ref. [41] derived the optimal protocol and its rate. However, no existing study addresses SPIR with multiple servers when classical MAC nor CQ MAC is given as a channel from servers to the user. In this paper, we focus on the case with two servers. That is, the quantum one-hop relay network model is given as follows. Two servers access the CQ MAC whose receiver is the user. The user has the respective point-to-point channels to both servers. This setting includes the case when the CQ MAC is a classical MAC.

As a preparation, we review the optimal method by Ref. [41] when noiseless classical channels from two servers to the user are available. Assume that we have $\mathrm{K}$ files and the $\mathrm{K}$ files messages are given as random variables $W_{1}, \ldots, W_{\mathrm{K}} \in \mathbb{F}_{p}^{\ell}$, which are shared by two servers. The two servers share uniform random variables $S \in \mathbb{F}_{p}^{\ell}$. The optimal protocol is the following when the user wants to get the $\theta$ th file.

Upload . The user generates $\mathrm{K}$ binary random variables $\left(Q_{1,1}, \ldots, Q_{1, \mathrm{~K}}\right) \in \mathbb{F}_{2}^{\mathrm{k}}$ independently subject to the uniform distribution. Then, the user sends the query $Q_{i}=$ $\left(Q_{i, 1}, \ldots, Q_{i, \mathrm{~K}}\right)$ to the $i$ th server, where $Q_{2, j}:=Q_{1, j}$ for $j \neq \theta$ and $Q_{2, \theta}:=Q_{1, \theta} \oplus 1$.

Download . The first server sends the user $X_{1}=$ $\left(\oplus_{j: Q_{1, j}=1} W_{j}\right) \oplus S$. The second server sends the user $X_{2}=$ $-\left[\left(\oplus_{j: Q_{2, j}=1} W_{j}\right) \oplus S\right]$.

Decoding . When $Q_{1, \theta}=1$, the user recovers the $\theta$ th file by $X_{1} \oplus X_{2}=W_{\theta}$. When $Q_{1, \theta}=0$, the user recovers the $\theta$ th file by $-\left(X_{1} \oplus X_{2}\right)=W_{\theta}$.
Combining the above idea with dense coding [45], the recent paper [41] addressed SPIR with multiple users with noiseless quantum channel.

Under the above protocol, the information for $\theta$ is not leaked to each server unless the two servers communicate each other. Also, the user cannot obtain any information for other files $W_{1}, \ldots, W_{\theta-1}, W_{\theta+1}, \ldots, W_{\mathrm{K}}$. Hence, the task for SPIR with two servers is realized by the above protocol. In the above protocol, the amount of upload information is a constant, which is independent of the file size.

Now, we consider the case when the quantum one-hop relay network model connects two servers and one user in the above way. Trivially, when we apply a SD code to a given CQ MAC, we can simulate the noiseless channel from each server. Hence, for the download step of we can use the noiseless channel realized by a SD code over a given CQ MAC. That is, in the download step, the $i$ th server sends $X_{i}$ to the user via the SD code over the given CQ MAC. This idea can be extended to the case with COMP codes. Since the key point of the above protocol is that the user recovers $X_{1} \oplus X_{2}$, we can apply our COMP code to the combination of the download step and the decoding step in the above protocol of SPIR with two servers. In summary, when a COMP code is employed to SPIR, the protocol in the download and decoding parts is changed as follows.

Download . The first server encodes $X_{1}=\left(\oplus_{j: Q_{1, j}=1} W_{j}\right) \oplus$ $S$, and the second server encodes $X_{2}=-\left[\left(\oplus_{j: Q_{2, j}=1} W_{j}\right) \oplus\right.$ $S$ ] by using a COMP code.

Decoding . Using a COMP code, the user decodes $X_{1} \oplus$ $X_{2}$. When $Q_{1, \theta}=1$, the user recovers the $\theta$ th file by $X_{1} \oplus$ 
$X_{2}=W_{\theta}$. When $Q_{1, \theta}=0$, the user recovers the $\theta$ th file by $-\left(X_{1} \oplus X_{2}\right)=W_{\theta}$.

Thus, when a CQ MAC is given and shared common random numbers between servers are allowed, the rate $C_{\text {COMP }}(\mathcal{W})$ is achievable for SPIR with two servers. Therefore, when we employ a SD code or a COMP code to realize SPIR with two servers, the rate of the SD code or the COMP code expresses the rate of SPIR based on the respective method. We observe that in-network computation (instead of simultaneous decoding) with COMP codes is enough to accomplish the networking task of private retrieval from a user to two servers.

\section{APPLICATION TO BUTTERFLY NETWORK}

As another example of the network topology, we can consider the butterfly network, shown in Fig. 5. While Refs. $[15,16]$ address it, they assume noiseless channels. In this network, node $v_{1}\left(v_{2}\right)$ intends to the message to node $v_{6}\left(v_{5}\right)$. The optimal method is presented in Fig. 5. To save time, it is natural that node $v_{3}$ receives the information from the two nodes $v_{1}$ and $v_{2}$ simultaneously, which implies that the channel to the node $v_{3}$ is a CQ MAC. Since node $v_{3}$ needs only the modulo sum of this information, we can apply our COMP code. This method can be applied to the channels to nodes $v_{5}$ and $v_{6}$ in the same way. That is, our COMP code can be applied 3 times in the butterfly network.

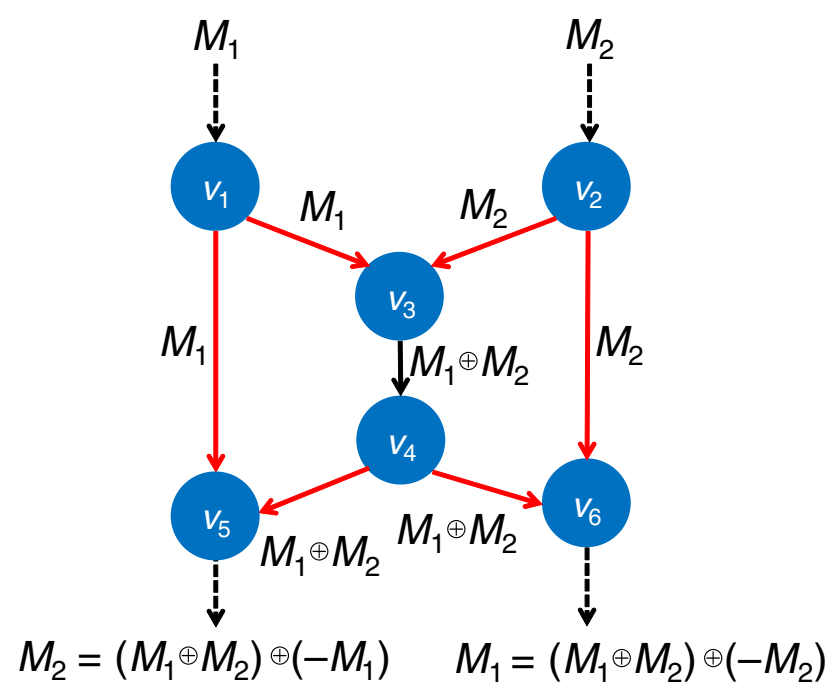

FIG. 5. Another example where COMP codes offer an advantage, the butterfly network. Node $v_{6}\left(v_{5}\right)$ intends to receive the message from node $v_{1}\left(v_{2}\right)$. In the optimal method, nodes $v_{3}$, $v_{5}$, and $v_{6}$ recover only the modulo sum of the two information messages. A red arrow shows a part of CQ MAC, in which our COMP code can be applied.

\section{DISCUSSION}

The general achievement of this paper is to show that the maximum achievable rate (capacity) requires codes reflecting the network topology of a quantum network when the quantum network has bottlenecks in the topology. As the main result of this paper, we introduce COMP codes over the CQ MAC, which is the simplest example of bottleneck in the physical topology, and derive general formulas of achievable rates.

To see the above fact in a physical concrete model, we demonstrate the effect of COMP codes over a bosonic CQ MAC whose quantum carriers are coherent states. Physically, a COMP code gathers photon power from interference by using the computation properties of the (quantized) electromagnetic field, rather than regarding interference as a noise. Under this model, we derive the analytical expressions and compute the numerical values of achievable rates for a practical discrete binary modulation, the binary phase-shift keying. Our results show that COMP codes achieve the transmission rate close to 1 under the above model at power $\|\alpha\| \approx 1$ while conventional codes cannot, which shows up to $33.33 \%$ gains over the conventional method. Surprisingly, not only with quantum detection, but also with suboptimal on-off and homodyne detection, COMP codes achieve the transmission rate close to 1 under the same model, just requiring more photon power than with collective measurement. Our results also show that the proposed method of COMP codes outperforms conventional codes only above a certain (small) threshold due to the quantum nature of the physical detection. That is, the quantum nature of the received signal offers an advantage for the decoding of the computation (sum) of the physical signal over SD codes only above such a threshold photon power while it does not below the threshold.

Finally, in order to clarify the applications, we find that while in traditional classical networks and the Internet, physical networks and their logical abstractions are purposely separately designed and managed (e.g., the former by engineers the latter by computer scientists), fully quantum networks are still in the pioneer days before establishing such a division between physical and logical parts. Hence, we explain two practical applications assuming seamless design of communication over the quantum network.

To simply clarify the advantage of COMP codes, this paper focuses on BPSK modulation on lossy coherent states as a simple modulation. Therefore, it is an interesting remaining study to investigate COMP codes with more practical modulations on coherent states over noisy bosonic channels.

While we prove that using computation properties of quantum interference can improve significantly the rates of a network, it seems reasonable to question whether such 
an improvement could be harnessed from other quantum resources. As an example, it is well known that preshared entanglement can significantly boost communication rates in the regime of high thermal noise and very low photon power [46-51]. This is due to the fact that entanglementassisted communication scales with the factor $\log \left(1 /|\alpha|^{2}\right)$ [50]. Hence, for the channel in our work, it is interesting to observe that while entanglement-assisted CQ MAC is useful only for very low photon power, COMP codes are useful only above a photon threshold, thus making both techniques compatible and complementary.

In addition, as another future problem, we can consider the secrecy condition for each message against the receiver. That is, we impose that the receiver can recover the modulo sum, but has no information for each message. Fortunately, the classical version of this problem has been studied in various research works [52-57]. Therefore, we can expect to generalize the above classical result to our quantum setting. Such a topic is another fruitful future study.

After our first submission of this work, we found a related paper, [58], which derived a result similar to Eq. (1) in Theorem 1. However, while our paper applies such result to realistic BPSK discrete modulation over a lossy bosonic network obtaining numerical results, the work [58] did not study any application.

\section{ACKNOWLEDGMENTS}

M.H. is supported in part by Guangdong Provincial Key Laboratory (Grant No. 2019B121203002).

\section{APPENDIX A: ANALYSIS ON AFFINE CODES}

\section{Optimal transmission rate with single sender}

As a preparation for our proof of Theorem 1 of the main body, we analyze affine codes over a classical-quantum channel $W=\left\{W_{x}\right\}_{x \in \mathcal{X}}$ from the classical alphabet $\mathcal{X}$ to the quantum system $\mathcal{H}_{Y}$, where $\mathcal{X}$ is a finite field $\mathbb{F}_{q}$ with prime power $q$. Here, $W_{x}$ for $x \in \mathcal{X}$ is a density matrix on $\mathcal{H}_{Y}$. The $n$-fold extension $W^{(n)}$ is defined so that $W_{x^{n}}^{(n)}$ is the product state $W_{x_{1}} \otimes \cdots \otimes W_{x_{n}}$ for $x^{n}=\left(x_{1}, \ldots, x_{n}\right) \in \mathcal{X}^{n}$. Let $I(X ; Y)_{\text {uni }}$ be the mutual information between $X$ and $Y$ when $X$ is subject to the uniform distribution.

In this section, for low-complexity construction of our codes, our encoder is limited to a code constructed by affine maps. A map $f$ onto a vector space is called affine when it is written as $f(x)=A(x) \oplus b$ with a linear map $A$ and a constant vector $b$.

Theorem 2. The mutual information $I(X ; Y)_{\text {uni }}$ equals the maximum transmission rate of an affine code with asymptotic zero error probability.
To show the achievability part of Theorem 2 , we randomly generate linear code with coding length $\ell$ as follows. We randomly choose a linear function $F$ from $\mathbb{F}_{q}^{\ell}$ to $\mathbb{F}_{q}^{n}$ such that

$$
\operatorname{Pr}\{x \in \operatorname{Im} F\} \leq q^{\ell-n} \text { for } x(\neq 0) \in \mathbb{F}_{q}^{n},
$$

where $\operatorname{Im} F$ expresses the image of the function of $F$. Such a construction of ensemble of linear function $F$ is given by using the Toeplitz matrix in Ref. [43]. We also randomly choose the shift vector $E \in \mathbb{F}_{p}^{n}$ subject to the uniform distribution as an variable independent of $F$. In the following, using $F$ and $E$, we define our affine code $G$ as $G(m):=F(m) \oplus E$. Then, we define our decoder for the code $G$ as follows. For $x^{n} \in \mid \mathcal{X}^{n}$, we define the projection

$$
\Pi_{x^{n}}:=\left\{W_{x^{n}}^{(n)} \geq q^{\ell} W_{\mathrm{mix}}^{\otimes n}\right\},
$$

where $W_{\text {mix }}:=\sum_{x \in \mathbb{F}_{q}}(1 / q) W_{x}$. Then, we define the decoder $\left\{D_{x^{n}, G}\right\}_{x^{n} \in \operatorname{Im} G}$

$$
D_{x^{n}, G}:=\left(\sum_{x^{n^{\prime}} \in \operatorname{Im} G} \Pi_{x^{n^{\prime}}}\right)^{-1 / 2} \Pi_{x^{n}}\left(\sum_{x^{n^{\prime} \in \operatorname{Im} G}} \Pi_{x^{n^{\prime}}}\right)^{-1 / 2} .
$$

We denote the decoding error probability of the code $G$ with the above decoder by $\epsilon(G)$.

Lemma 1. When we generated our affine code $G$ as the above, the average of the decoding error probability $\epsilon(G)$ is upper bounded as

$$
\mathbb{E}_{G} \epsilon(G) \leq 6 q^{s \ell} e^{n E(s)},
$$

with $s \in(0,1)$ where $E(s):=\log \left(\sum_{x \in \mathbb{F}_{q}} q^{-1} \operatorname{Tr} W_{x}^{1-s} W_{\text {mix }}^{s}\right)$.

This proposition shows the achievability part of Proposition 2 because $E(s) \cong s I(X ; Y)_{\text {uni }}$ when $s$ is close to 0 .

Conversely, the impossibility part of Theorem 2 can be shown as follows. Let $g$ be an affine code with block length $n$. Assume $M$ is the message subject to the uniform distribution and $X^{n}=\left(X_{1}, \ldots, X_{n}\right)$ is given as $g(M)$. The mutual information $I\left(X^{n} ; Y^{n}\right)$ is evaluated by

$$
\begin{aligned}
I\left(X^{n} ; Y^{n}\right) & =\sum_{i=1}^{n} I\left(X^{n} ; Y_{i} \mid Y_{1}, \ldots, Y_{i-1}\right) \\
& \leq \sum_{i=1}^{n} I\left(X^{n}, Y_{1}, \ldots, Y_{i-1} ; Y_{i}\right)=\sum_{i=1}^{n} I\left(X_{i} ; Y_{i}\right)
\end{aligned}
$$

because of the Markov chain $\left(X^{n}, Y_{1}, \ldots, Y_{i-1}\right)-X_{i}-Y_{i}$. Since $g$ is an affine map, $X_{i}$ is subject to the uniform distribution unless it is a constant. Hence, $I\left(X_{i} ; Y_{i}\right)$ is upper 
bounded by $I(X ; Y)_{\text {uni. }}$. Combining the Fano inequality, we can show the above fact.

\section{Proof of Lemma 1}

Lemma 1 can be shown as follows. Assume the following condition for our code $G$ :

$\mathrm{C}-x^{n}$ The element $x^{n} \in \mathcal{X}^{n}$ is chosen as a code word of the code $G$.

Then, the condition, Eq. (A6), yields the following inequality for $x^{n^{\prime}}\left(\neq x^{n}\right) \in \mathbb{F}_{q}^{n}$ :

$$
\operatorname{Pr}_{C-x^{n}}\left\{x^{n^{\prime}} \in \operatorname{Im} G\right\} \leq q^{\ell-n},
$$

where $\operatorname{Pr}_{C-x^{n}}$ expresses the conditional probability with the condition $C-x^{n}$.

By using Hayashi-Nagaoka inequality [42], the decoding error probability for $x^{n} \in \mathcal{X}^{n}$ is evaluated as

$$
\begin{aligned}
\operatorname{Tr} W_{x^{n}}^{(n)}\left(I-D_{x^{n}, G}\right) \leq & 2 \operatorname{Tr} W_{x^{n}}^{(n)}\left(I-\Pi_{x^{n}}\right) \\
& +4 \operatorname{Tr} W_{x^{n}}^{(n)}\left(\sum_{x^{n^{\prime}}\left(\neq x^{n}\right) \in \operatorname{Im} G} \Pi_{x^{n^{\prime}}}\right) .
\end{aligned}
$$

Then,

$$
\begin{aligned}
\mathbb{E}_{G} & {\left[\sum_{x^{n} \in \operatorname{Im} G} q^{-\ell} \operatorname{Tr} W_{x^{n}}^{(n)}\left(I-\Pi_{x^{n}}\right)\right] } \\
\leq & \mathbb{E}_{G}\left[\sum_{x^{n} \in \operatorname{Im} G} q^{-\ell} \operatorname{Tr}\left(W_{x^{n}}^{(n)}\right)^{1-s} q^{s \ell}\left(W_{\text {mix }}^{\otimes n}\right)^{s}\right. \\
& =q^{s \ell}\left(\sum_{x \in \mathbb{F}_{q}} q^{-1} \operatorname{Tr} W_{x}^{1-s} W_{\text {mix }}^{s}\right)^{n}=q^{s \ell} e^{n E(s)} .
\end{aligned}
$$

Hence, we have

$$
\begin{aligned}
\mathbb{E}_{G} & {\left[\sum_{x^{n} \in \operatorname{Im} G} q^{-\ell} \operatorname{Tr} W_{x^{n}}^{(n)}\left(\sum_{x^{n^{\prime}}\left(\neq x^{n}\right) \in \operatorname{Im} G} \Pi_{x^{n^{\prime}}}\right)\right], } \\
= & \mathbb{E}_{G}\left[q^{-\ell} \sum_{x^{n^{\prime} \in \operatorname{Im} G}} \operatorname{Tr}_{x^{n^{\prime}}}\left(\sum_{x^{n}\left(\neq x^{n^{\prime}}\right) \in \operatorname{Im} G} W_{x^{n}}^{(n)}\right)\right], \\
= & \mathbb{E}_{G}\left[q^{-\ell} \sum_{x^{n^{\prime}} \in \operatorname{Im} G} \operatorname{Tr} \Pi_{x^{n^{\prime}}} \mathbb{E}_{G \mid C-x^{n}}\left(\sum_{x^{n}\left(\neq x^{n^{\prime}}\right) \in \operatorname{Im} G} W_{x^{n}}^{(n)}\right)\right], \\
= & \mathbb{E}_{G}\left[q^{-\ell} \sum_{x^{n^{\prime} \in \operatorname{Im} G}} \operatorname{Tr} \Pi_{x^{n^{\prime}}}\right. \\
& \left.\left(\sum_{x^{n}\left(\neq x^{n^{\prime}}\right) \in \mathbb{F}_{q}^{n}} \operatorname{Pr}_{C-x^{n}}\left\{x^{n^{\prime}} \in \operatorname{Im} G\right\} W_{x^{n}}^{(n)}\right)\right], \\
\leq & \mathbb{E}_{G}\left[q^{-\ell} \sum_{x^{n^{\prime} \in \operatorname{Im} G}} \operatorname{Tr}_{x^{n^{\prime}}}\left(\sum_{x^{n}\left(\neq x^{n^{\prime}}\right) \in \mathbb{F}_{q}^{n}} q^{\ell-n} W_{x^{n}}^{(n)}\right)\right],
\end{aligned}
$$

$$
\begin{aligned}
& \leq \mathbb{E}_{G}\left[q^{-n} \sum_{x^{n^{\prime}} \in \operatorname{Im} G} \operatorname{Tr}_{x^{n^{\prime}}}\left(\sum_{x^{n} \in \mathbb{F}_{q}^{n}} W_{x^{n}}^{(n)}\right)\right], \\
& =\mathbb{E}_{G}\left[q^{-n} \sum_{x^{n^{\prime}} \in \operatorname{Im} G} \operatorname{Tr} \Pi_{x^{n^{\prime}}} q^{n} W_{\mathrm{mix}}^{\otimes n}\right], \\
& \leq \mathbb{E}_{G}\left[\sum_{x^{n^{\prime}} \in \operatorname{Im} G} \operatorname{Tr}\left(W_{x^{n^{\prime}}}^{(n)}\right)^{1-s} q^{(1-s) \ell}\left(W_{\mathrm{mix}}^{\otimes n}\right)^{s}\right], \\
& =q^{\ell-n} \sum_{x^{n^{\prime} \in \mathbb{F}_{q}^{n}}} \operatorname{Tr}\left(W_{x^{n^{\prime}}}^{(n)}\right)^{1-s} q^{(1-s) \ell}\left(W_{\mathrm{mix}}^{\otimes n}\right)^{s}, \\
& =q^{s \ell}\left(\sum_{x \in \mathbb{F}_{q}} q^{-1} \operatorname{Tr} W_{x}^{1-s} W_{\mathrm{mix}}^{s}\right)^{n}=q^{s \ell} e^{n E(s)} .
\end{aligned}
$$

Therefore, the average of the decoding error probability is evaluated as

$$
\mathbb{E}_{G} \operatorname{Tr} W_{x^{n}}^{(n)}\left(I-D_{x^{n}, G}\right) \leq 6 q^{s \ell} e^{n E(s)} .
$$

we obtain Lemma 1.

\section{APPENDIX B: PROOF OF THEOREM 1}

\section{Lower bound}

First, generalizing the method of Ref. [36] for classical channels to classical-quantum MACs, we prove the lower bound (1) of the main body. That is, we show that the mutual information rate $I(Y ; A \oplus B)$ can be achieved in the sense of computation and forward. To show this theorem, we define the degraded channel $\left\{W_{x \mid d}\right\}_{x \in \mathbb{F}_{p}}$ from $\mathbb{F}_{p}$ to $\mathcal{H}_{Y}$ as $W_{x}^{d}:=\sum_{x^{\prime} \in \mathbb{F}_{p}} p^{-1} W_{x^{\prime}, x \ominus x}$ for $x \in \mathbb{F}_{p}$. When $X$ is subject to the uniform distribution on $\mathbb{F}_{p}$ and the output state is given as $W_{X \mid d}$, we have $I(Y ; A \oplus B)_{\text {uni }}=I(Y ; X)$.

We randomly generate our code with coding length $\ell$. We randomly choose a linear function $F$ as Eq. (A6) in the same way as Sec. 1 . We extend the function $F$ to an invertible linear function $\bar{F}$ on $\mathbb{F}_{p}^{n}$ such that the restriction of $\bar{F}$ to the first $\ell$ component equals the function $F$. We also randomly choose the shift vectors $E \in \mathbb{F}_{p}^{n}$ and $E^{\prime} \in$ $\mathbb{F}_{p}^{n-\ell}$ independently subject to the uniform distribution as other variables. In the following, using $\bar{F}, E$, and $E^{\prime}$, we construct our code as follows. That is, our code depends on $\bar{F}, E$, and $E^{\prime}$. Also, we define the affine code $G$ as $G(m):=$ $F(m) \oplus E$.

The sender $A$ encodes the message $M_{A}^{n}$ to $\bar{F}\left(M_{A}^{n}, E^{\prime}\right)$, and the sender $B$ encodes the message $M_{B}^{n}$ to $\bar{F}\left(M_{B}^{n},-E^{\prime}\right) \oplus E$. In the following, we assume that the receiver makes the decoder dependent only on $\bar{F}, F$, and $E$. Hence, $\bar{F}, F, E$ are fixed to $\bar{f}, f$, and $e$. Also, $g$ express the affine code defined as $g(m):=f(m) \oplus e$. 
When $M_{A}^{n} \oplus M_{B}^{n}=m$, the output state is

$$
\begin{aligned}
& \mathbb{E}_{M_{A}^{n}, E^{\prime}} W_{\bar{f}\left(M_{A}^{n}, E^{\prime}\right) \bar{f}\left(m \ominus M_{A}^{n},-E^{\prime}\right) \oplus e}^{(n)} \\
& =\mathbb{E}_{M_{A}^{n}, E^{\prime}} W_{\bar{f}\left(M_{A}^{n}, E^{\prime}\right) \bar{f}(m) \oplus e \ominus \bar{f}\left(M_{A}^{n}, E^{\prime}\right)}^{(n)} \\
& =q^{-n} \sum_{x^{n} \in \mathbb{F}_{p}^{n}} W_{x^{n}, \bar{f}(m) \oplus e \ominus x^{n}}^{(n)}=W_{\bar{f}(m) \oplus e}^{d,(n)}=W_{f(m) \oplus e}^{d,(n)} .
\end{aligned}
$$

Hence, the above state is the output state with the following case. The encoder is the affine code $g$ and the channel is the degraded channel $W^{d}$. Given the affine code $g$, we choose the decoder based on Eq. (A3) when the channel is the degraded channel $W^{d}$.

Now, we consider the case when $F$ and $E$ are randomly chosen in the above way. This random choice of $G$ is the same as Lemma 1. Hence, Lemma 1 guarantees that the rate $I(Y ; A \oplus B)_{\text {uni }}=I(Y ; X)$ is achievable, which implies (1) of the main body.

\section{Upper bound}

Next, we prove the upper bound (2) of the main body. For this aim, we focus on a sequence of codes $\left\{\Psi_{n}\right\}$ with a transmission rate pair $R$, where the pair of encoders $\left(\phi_{A, n}, \phi_{B, n}\right)$ of $\Psi_{n}$ satisfies the following: $\phi_{A, n}\left(\phi_{B, n}\right)$ maps $M_{A, n}\left(M_{B, n}\right)$ to $X_{A}^{n}=\left(X_{A, i}\right)_{i=1}^{n}\left[X_{B}^{n}=\left(X_{B, i}\right)_{i=1}^{n}\right]$. Here, $M_{A, n}$ and $M_{B, n}$ are independently subject to the uniform distribution. Since

$$
\begin{aligned}
& I\left(M_{A, n} \oplus M_{B, n} ; Y^{n} \mid M_{A, n}\right) \\
& \quad=I\left(M_{A, n} \oplus M_{B, n} ; Y^{n} M_{A, n}\right)-I\left(M_{A, n} \oplus M_{B, n} ; M_{A, n}\right) \\
& \quad=I\left(M_{A, n} \oplus M_{B, n} ; Y^{n} M_{A, n}\right) \geq I\left(M_{A, n} \oplus M_{B, n} ; Y^{n}\right),
\end{aligned}
$$

we find that

$$
\begin{aligned}
& I\left(M_{A, n} \oplus M_{B, n} ; Y^{n}\right) \leq\left(M_{A, n} \oplus M_{B, n} ; Y^{n} \mid M_{A, n}\right) \\
& \quad=I\left(M_{B, n} ; Y^{n} \mid M_{A, n}\right)=I\left(X_{B}^{n} ; Y^{n} \mid X_{A}^{n}\right) \\
& \quad=\sum_{i=1}^{n} I\left(X_{B}^{n} ; Y_{i} \mid Y^{i-1} X_{A}^{n}\right)=\sum_{i=1}^{n} I\left(X_{B, i} ; Y_{i} \mid Y^{i-1} X_{A}^{n}\right) \\
& \quad \stackrel{(a)}{\leq} \sum_{i=1}^{n} I\left(X_{B, i} ; Y_{i} \mid X_{A}^{n}\right),=\sum_{i=1}^{n} I\left(X_{B, i} ; Y_{i} \mid X_{A, i}\right)
\end{aligned}
$$

where (a) follows from the Markov chain $Y^{i-1}-X_{B, i}-Y_{i}$ when $X_{A}^{n}$ is fixed.

Combining Fano's inequality, we can show that

$$
R \leq \max _{P_{A}, P_{B}} I(B ; Y \mid A)_{P_{A} \times P_{B}}=\max _{P_{B}, a \in \mathbb{F}_{p}} I(B ; Y \mid A=a)_{P_{B}} .
$$

In the same way, we can show

$$
R \leq \max _{P_{A}, P_{B}} I(A ; Y \mid B)_{P_{A} \times P_{B}}=\max _{P_{A}, b \in \mathbb{F}_{p}} I(A ; Y \mid B=b)_{P_{A}} .
$$

[1] H.-Y. Liu et al., Optical-Relayed Entanglement Distribution Using Drones as Mobile Nodes, Phys. Rev. Lett. 126, 020503 (2021).

[2] S. Pirandola, R. Laurenza, C. Ottaviani, and L. Banchi, Fundamental limits of repeaterless quantum communications, Nat. Commun. 8, 15043 (2017).

[3] K. Azuma, A. Mizutani, and H. K. Lo, Fundamental rateloss trade-off for the quantum internet, Nat. Commun. 7, 13523 (2016).

[4] Y.-A. Chen, Q. Zhang, T.-Y. Chen, W.-Q. Cai, S.-K. Liao, J. Zhang, K. Chen, J. Yin, J.-G. Ren, and Z. Chen et al., An integrated space-to-ground quantum communication network over 4, 600 kilometres, Nature 589, 214 (2021).

[5] J. F. Dynes, A. Wonfor, W. W.-S. Tam, A. W. Sharpe, R. Takahashi, M. Lucamarini, A. Plews, Z. L. Yuan, A. R. Dixon, J. Cho, and Y. Tanizawa, et al., Cambridge quantum network, npj Quantum Inf. 5, 101 (2019).

[6] C. Elliott, Building the quantum network, New J. Phys. 4, 46 (2002).

[7] S. K. Joshi, D. Aktas, S. Wengerowsky, F. Loncaric, S. P. Neumann, B. Liu, T. Scheidl, Z. Samec, L. Kling, and A. Qiu, et al., A trusted node-free eight-user metropolitan quantum communication network, Sci. Adv. 6 (2020).

[8] H. J. Kimble, The quantum internet, Nature 453, 1023 (2008).

[9] A. Reiserer and G. Rempe, Cavity-based quantum networks with single atoms and optical photons, Rev. Mod. Phys. 87, 1379 (2015).

[10] S. Wehner, D. Elkouss, and R. Hanson, Quantum internet: A vision for the road ahead, Science 362, 9288 (2018).

[11] T. Satoh, K. Ishizaki, S. Nagayama, and R. Van Meter, Analysis of quantum network coding for realistic repeater networks, Phys. Rev. A 93, 032302 (2016).

[12] S. J. Devitt, A. D. Greentree, A. M. Stephens, and R. Van Meter, High-speed quantum networking by ship, Sci. Rep. 6, 36163 (2016).

[13] A. Winter, The capacity of the quantum multiple access channel, IEEE Trans. Inf. Theory 47, 3059 (2001).

[14] R. Ahlswede, N. Cai, S.-Y. R. Li, and R. W. Yeung, Network information flow, IEEE Trans. Inf. Theory 46, 1204 (2000).

[15] M. Hayashi, K. Iwama, H. Nishimura, R. Raymond, and S. Yamashita, in STACS 2007 SE - 52, edited by W. Thomas and P. Weil, Lecture Notes in Computer Science Vol. 4393 (Springer, Berlin Heidelberg, 2007).

[16] M. Hayashi, Prior entanglement between senders enables perfect quantum network coding with modification, Phys. Rev. A 76, 40301 (2007).

[17] H. Kobayashi, F. Le Gall, H. Nishimura, and M. Rötteler, in Automata, Languages and Programming, edited by S. Albers, A. Marchetti-Spaccamela, Y. Matias, S. Nikoletseas, and W. Thomas, Lecture Notes in Computer Science Vol. 5555 (Springer, Berlin Heidelberg, 2009). 
[18] D. Leung, J. Oppenheim, and A. Winter, Quantum network communication; the butterfly and beyond, IEEE Trans. Inf. Theory 56, 3478 (2010).

[19] H. Kobayashi, F. Le Gall, H. Nishimura, and M. Rotteler, in Proceedings of IEEE International Symposium on Information Theory (IEEE, Austin, 2010).

[20] H. Kobayashi, F. Le Gall, H. Nishimura, and M. Rotteler, in Proceedings of IEEE International Symposium on Information Theory (IEEE, St. Petersburg, 2011).

[21] A. Jain, M. Franceschetti, and D. A. Meyer, On quantum network coding, J. Math. Phys. 52, 032201 (2011).

[22] M. Owari, G. Kato, and M. Hayashi, Secure quantum network coding on butterfly network, Quantum Sci. Technol. 3, 014001 (2017).

[23] G. Kato, M. Owari, and M. Hayashi, in 10th International Conference on Information Theoretic Security, editor J. Shikata, Lecture Notes in Computer Science Vol. 10681 (Springer, Hong Kong, 2017).

[24] H. Lu, Z. Li, X. Yin, R. Zhang, X. Fang, L. Li, N. Liu, F. $\mathrm{Xu}, \mathrm{Y}$. Chen, and J. Pan, Experimental quantum network coding, npj Quantum Inf. 5, 89 (2019).

[25] S. Song and M. Hayashi, Secure quantum network code without classical communication, IEEE Trans. Inf. Theory 66, 1178 (2020).

[26] B. J. Yen and J. H. Shapiro, in Proceedings of SPIE 5842, Fluctuations and Noise in Photonics and Quantum Optics III (IEEE, Austin, 2005).

[27] M. Wilde and S. Guha, in Proceedings of International Symposium on Information Theory and its Applications (IEEE, Cambridge, USA, 2012).

[28] S. Zhang, S. C. Liew, and P. P. Lam, in Proceedings of ACM Mobile Computing and Networking (Los Angeles, 2006).

[29] B. Nazer and M. Gastpar, in Proceedings of IEEE International Symposium on Information Theory (IEEE, Istanbul, 2006).

[30] P. Popovski and H. Yomo, in Proceedings of IEEE International Conference on Communications (IEEE, Istanbul, 2006).

[31] B. A. Nazer and M. Gastpar, Computation over multipleaccess channels, IEEE Trans. Inf. Theory 53, 3498 (2007).

[32] B. Nazer and M. Gastpar, in Proceedings of Asilomar Conference on Signals, Systems and Computers (IEEE, Pacific Grove, 2008).

[33] B. Nazer and M. Gastpar, Compute-and-forward: Harnessing interference through structured codes, IEEE Trans. Inf. Theory 57, 6463 (2011).

[34] B. Nazer and M. Gastpar, Reliable physical layer network coding, Proc. IEEE 99, 438 (2011).

[35] B. Nazer, V. R. Cadambe, V. Ntranos, and G. Caire, Expanding the compute-and-forward framework: Unequal powers, signal levels, and multiple linear combinations, IEEE Trans. Inf. Theory 62, 4879 (2016).

[36] S. S. Ullah, G. Liva, and S. C. Liew, in Proceedings of the IEEE Information Theory Workshop (IEEE, Kaohsiung, 2017).

[37] S. Takabe, T. Wadayama, and M. Hayashi, in Proceedings of IEEE International Symposium on Information Theory (IEEE, Paris, 2019).

[38] S. Takabe and T. Wadayama and M. Hayashi, Asymptotic behavior of spatial coupling LDPC coding for computeand-forward two-way relaying, IEEE Trans. Comm. 68, 4063 (2020).
[39] M. Hayashi and Á. Vázquez-Castro, Physical layer computation as NOMA for integrated wireless systems, IEEE Trans. Comm. 69, 4520 (2021).

[40] H. Sun and S. A. Jafar, The capacity of symmetric private information retrieval, IEEE Trans. Inf. Theory $\mathbf{6 5}, 322$ (2019).

[41] S. Song and M. Hayashi, Capacity of quantum private information retrieval with multiple servers, IEEE Trans. Inf. Theory 67, 452 (2021).

[42] M. Hayashi and H. Nagaoka, General formulas for capacity of classical-quantum channels, IEEE Trans. Inf. Theory 49, 1753 (2003).

[43] T. Tsurumaru and M. Hayashi, Dual universality of hash functions and its applications to quantum cryptography, IEEE Trans. Inf. Theory 59, 4700 (2013).

[44] H. Shi, M.-H. Hsieh, S. Guha, Z. Zhang, and Q. Zhuang, Entanglement-assisted multiple-access channels: Capacity regions and protocol designs, npj Quantum Inf. 7, 74 (2021).

[45] C. H. Bennett and S. J. Wiesner, Communication via oneand Two-Particle Operators on Einstein-Podolsky-Rosen States, Phys. Rev. Lett. 69, 2881 (1992).

[46] C. H. Bennett, P. W. Shor, J. A. Smolin, and A. V. Thapliyal, Entanglement-assisted capacity of a quantum channel and the reverse Shannon theorem, IEEE Trans. Inf. Theory 48, 2637 (2002).

[47] A. S. Holevo, On entanglement-assisted classical capacity, J. Math. Phys. 43, 4326 (2002).

[48] M.-H. Hsieh, I. Devetak, and A. Winter, Entanglement assisted capacity of quantum multiple-access channels, IEEE Trans. Inf. Theory 54, 3078 (2008).

[49] H. Shi, Z. Zhang, and Q. Zhuang, Practical Route to Entanglement-Assisted Communication Over Noisy Bosonic Channels, Phys. Rev. Appl. 13, 034029 (2020).

[50] S. Guha, Q. Zhuang, and B. A. Bash, in Proceedings of IEEE International Symposium on Information Theory (2020).

[51] K. Wang and M. Hayashi, Permutation enhances classical communication assisted by entangled states, IEEE Trans. Inf. Theory 67, 3905 (2021).

[52] Z. Ren, J. Goseling, J. H. Weber, and M. Gastpar, in Proceedings of IEEE Information Theory Workshop (IEEE, Jerusalem, 2015).

[53] X. He and A. Yener, End-to-end secure multi-hop communication with untrusted relays, IEEE Trans. Wireless Comm. 12, 1 (2013).

[54] X. He and A. Yener, Strong secrecy and reliable byzantine detection in the presence of an untrusted relay, IEEE Trans. Inf. Theory 59, 177 (2013).

[55] S. Vatedka, N. Kashyap, and A. Thangaraj, Secure compute-and-forward in a bidirectional relay, IEEE Trans. Inf. Theory 61, 2531 (2015).

[56] A. A. Zewail and A. Yener, in Proceedings of IEEE Information Theory Workshop (IEEE, Jerusalem, 2015).

[57] M. Hayashi, T. Wadayama, and Á. Vázquez-Castro, Secure computation-and-forward with linear codes, IEEE J. Sel. Areas Inf. Theory 2, 139 (2021).

[58] T. A. Atif, A. Padakandla, and S. S. Pradhan, in Proceedings of IEEE International Symposium on Information Theory (IEEE, Melbourne, 2021). Available at ArXiv:2103.02082. 